\section{Condições de trabalho e automedicação em profissionais da rede básica de saúde da zona urbana de Pelotas, RS}

\section{Working conditions and self- \\ medication among primary healthcare professionals in an urban area of Pelotas, $R S$}

\section{Elaine Tomasi ${ }^{1}$}

\section{Graciela Castro Sant'Anna²}

Ana Maria Oppelt²

Raquel Magalhães Petrini

Inês Vianna Pereira²

Bárbara Tomasi Sassi ${ }^{3}$

'Escola de Psicologia, Universidade Católica de Pelotas

${ }^{2}$ Acadêmicas de Psicologia - Universidade Católica de Pelotas

${ }^{3}$ Acadêmica de Medicina - Universidade Federal de Pelotas

Correspondência: Elaine Tomasi. Rua Marechal Deodoro, 1078/304 Pelotas, RS. CEP 96.020-220. Email: tomasiet@uol.com.br

\section{Resumo}

Este estudo transversal investigou condições de trabalho e a morbidade dos profissionais de saúde da atenção básica em Pelotas, através de informações sociodemográficas, comportamentais, da atividade e ambiente de trabalho e de morbidade. Foram estudados 329 profissionais em 39 serviços. A grande maioria era do sexo feminino $(80 \%)$ e a média de idade era de 41 anos ( $\pm 9,2$ anos). Quase $30 \%$ dos profissionais pertenciam às classes $\mathrm{C}$ e $\mathrm{D}$. O hábito de fumar foi referido por $19 \%$ dos indivíduos da amostra e $61 \%$ não praticaram nenhuma atividade física regular no mês anterior à entrevista. Cerca de metade dos entrevistados tinha outro emprego (51\%) e $25 \%$ precisaram faltar ao trabalho no último mês, principalmente por problemas pessoais de saúde (59\%) e problemas familiares (22\%). Em média, os trabalhadores atendiam cerca de 30 pessoas por dia em uma jornada semanal de 40 horas. Problemas de saúde foram referidos por $40 \%$ dos entrevistados, com destaque para problemas do aparelho circulatório (27\%) e osteomusculares (18\%). Independente de ter problema de saúde, $67 \%$ dos entrevistados faziam uso de medicamentos regularmente e $47 \%$ referiram este uso nos últimos 15 dias. Um quarto costumava automedicar-se, significativamente em maior proporção entre médicos e outros profissionais de nível superior, entre os trabalhadores de maior nível socioeconômico e entre aqueles com mais de um emprego.

Palavras-chave: Profissionais de saúde. Automedicação. Saúde do trabalhador. 


\section{Abstract}

This cross-sectional study investigated working conditions and the morbidity of basic healthcare professionals in Pelotas using data on sociodemographics, behavior, activities and working environment, as well as morbidity. 329 professionals from 39 healthcare services were studied. The vast majority of these were women (80\%) and the average age was 41 ( \pm 9.2 years). Almost $30 \%$ of these professionals came from the $\mathrm{C}$ and D social strata; $19 \%$ were smokers and $61 \%$ had not done any regular physical exercise during the one month prior to being interviewed. Almost half of the interviewees had another job (51\%) and $25 \%$ needed to miss work over the past month mainly due to personal health problems $(59 \%)$ and to family problems (22\%). On average, workers saw nearly 30 people per day in a 40 -hour working week. Health problems were reported by $40 \%$ of the interviewees, $27 \%$ concerning circulation, and $18 \%$ concerning osteomuscular difficulties. Apart from having health problems, $67 \%$ of the interviewees used medicines on a regular basis and $47 \%$ had used medicines in the past 15 days. A quarter were accustomed to self-medicating themselves, with a significantly greater proportion among doctors and other professionals of a higher level, among workers of greater socioeconomic status and among those with more than one job.

Keywords: Healthcare professionals. Selfmedication. Worker health.

\section{Introdução}

A partir da década de 90, a implantação e a consolidação do Sistema Único de Saúde (SUS) vêm desafiando trabalhadores e gestores na condução e aperfeiçoamento do sistema como um todo. Os preceitos básicos incluem a universalidade e eqüidade no acesso à atenção em saúde, um modelo assistencial com ênfase na integralidade das ações, o direito à informação, o controle social, a regionalização e a hierarquização das ações ${ }^{1}$.

As expectativas da sociedade por melhores serviços de saúde têm crescido desde então, com grande destaque para a rede básica como porta de entrada dos usuários no sistema público de saúde. É consenso que o SUS deva ser cada vez mais justo e eficiente, gastando melhor os recursos disponíveis e fazendo mais e melhor pela saúde da população, qualificando as estruturas e principalmente os processos da atenção à saúde.

Em saúde pública, um dos aspectos menos estudados na avaliação da qualidade da atenção diz respeito aos cuidadores, ou seja, àqueles profissionais que são responsáveis pelo atendimento direto dos usuários de serviços - médicos, enfermeiros, dentistas, assistentes sociais, psicólogos, nutricionistas, técnicos e auxiliares de saúde, agentes comunitários e administrativos.

Acredita-se que estes profissionais constituem um grupo de trabalhadores cujo processo de trabalho é bastante peculiar, onde interagem habilidades técnicas e relações interpessoais, além do compromisso implícito, e para muitos desconhecido, com o entender coletivo do processo saúde-doença, recaindo sobre eles grandes e crescentes responsabilidades.

O impacto do trabalho sobre a saúde tem sido investigado com regularidade em diversas categorias profissionais ${ }^{2-4}$. A ocorrência de acidentes de trabalho envolvendo exposição a sangue e secreções corporais são comuns, particularmente entre trabalhadores de estabelecimentos hospitalares ${ }^{5}$. 
Diversos fatores têm sido relacionados ao estresse ocupacional em diferentes atividades produtivas, com destaque para o trabalho por turnos, o trabalho noturno, a sobrecarga quantitativa e qualitativa de trabalho, a falta de controle sobre as atividades, a remuneração, a responsabilidade excessiva, a exposição a situações de enfrentamento, o trabalho rotineiro, a qualidade das relações interpessoais, a falta de segurança e a instabilidade no emprego. Discussões a respeito da equipe multiprofissional para atenção básica à saúde consideram divisão do trabalho, status da profissão, posição no processo de trabalho, aspectos organizacionais, relações informais, redes de poder, valores e normas como fatores relacionados ao desempenho do trabalho ${ }^{6}$.

Amaral $^{7}$, avaliando a implantação de serviços de saúde mental na atenção básica, destaca que a definição dos papéis dentro das equipes, em geral manteve a divisão tradicional entre o trabalho do médico e dos não-médicos. O baixo envolvimento das equipes com o funcionamento global das unidades e a articulação entre as áreas clínicas prejudicaram a atenção integral aos problemas de saúde dos usuários.

De acordo com Paulo \& Zanine ${ }^{8}$, “a automedicação é um procedimento caracterizado fundamentalmente pela iniciativa de um doente, ou de seu responsável, em obter ou produzir e utilizar um produto que acredita lhe trará benefícios no tratamento de doenças ou alívio de sintomas”. A automedicação inadequada, tal como a prescrição errônea, pode ter como conseqüência efeitos indesejáveis, enfermidades iatrogênicas e mascaramento de doenças evolutivas, representando, portanto, um problema a ser prevenido. É evidente que o risco dessa prática está correlacionado com o grau de instrução e informação dos usuários sobre medicamentos, bem como com a acessibilidade dos mesmos ao sistema de saúde.

O objetivo deste estudo foi elaborar um diagnóstico das condições de trabalho e saúde - física e mental - de profissionais que atendem a população de Pelotas em unidades básicas, com ênfase na automedicação. Acredita-se que este conhecimento possa subsidiar projetos de intervenção e programas de capacitação com vistas à melhoria das condições de trabalho e saúde destes profissionais e, conseqüentemente, da população em geral.

\section{Métodos}

O estudo foi do tipo transversal, com base em todos os serviços de saúde de atenção básica da zona urbana de Pelotas. Entre os meses de maio a setembro de 2004, buscou-se entrevistar todos os profissionais que desempenhavam tarefas de atenção à saúde e entravam em contato direto com a população nas 39 unidades de saúde da zona urbana. Os dados foram coletados através de questionário padronizado e précodificado, aplicado no próprio local de trabalho por alunos da Escola de Psicologia da UCPEL e da Faculdade de Medicina da UFPEL, previamente capacitados. Este questionário continha informações:

- sociodemográficas: sexo, idade, escolaridade, nível socioeconômico (Associação Brasileira de Institutos de Pesquisa e Mercado - ABIPEME);

- condições de trabalho: função, tempo de trabalho total e na instituição, outros vínculos empregatícios, carga horária, absenteísmo, atendimentos/período, estressores psicossociais no ambiente de trabalho ${ }^{9}$ - divididos em quatro aspectos: do ambiente físico, das tarefas, das relações institucionais e das relações pessoais;

- estilo de vida e morbidade: hábito de fumar, atividade física, problemas de saúde, incluindo depressão - aferida pelo Beck Depression Inventory (BDI) ${ }^{10}$ -, utilização de serviços de saúde e uso de medicamentos sem prescrição médica. Os estressores psicossociais foram analisados a partir da mediana dos escores obtidos para cada um dos aspectos. 
O estudo foi aprovado pelo Comitê de Ética da Universidade Federal do Sul (UFRGS) e a todos os entrevistados foi garantido o direito de não participação e o sigilo das informações; aos que concordaram em responder o questionário foi solicitada assinatura de termo de consentimento.

Os questionários foram revisados, codificados e digitados dupla e independentemente para verificação de erros no programa EPIINFO 6.04.d. O banco de dados final foi convertido para o pacote estatístico SPSS, onde as variáveis receberam o primeiro tratamento, incluindo sua rotulação e checagem de amplitude e consistência. Em seguida, foram realizadas as análises bivariadas, comparando-se a prevalência de automedicação de acordo com as variáveis demográficas e ocupacionais. Para isso, foram calculadas as razões de prevalência e seus respectivos intervalos de confiança de $95 \%$.

\section{Resultados}

Foram estudados 329 profissionais, distribuídos em 39 unidades de saúde, sendo 32 unidades básicas de saúde (UBS) e sete Centros de Atenção Psicossocial (CAPs). O número estimado de trabalhadores era de 400, o que implicou em um percentual de perdas e recusas de $18 \%$, a maioria dos quais referindo-se a profissionais que, alegando falta de tempo, recusaram-se a participar e responder ao questionário (13\%). Também foram registrados casos de trabalhadores em férias ou licença (5\%). A grande maioria dos profissionais de saúde entrevistados era do sexo feminino (80\%), e a média de idade foi de 41 anos (desvio-padrão de 9,2 anos). Cerca de $8 \%$ tinham menos que o segundo grau de escolaridade e um terço havia feito pósgraduação. De acordo com a classificação da ABIPEME, quase $30 \%$ dos profissionais pertencia às classes $\mathrm{C} \mathrm{e} \mathrm{D}$.

Do total da amostra, $80 \%$ trabalhavam em UBS e $20 \%$ em Centros de Atenção Psicossocial (CAPs). A Figura 1 apresenta a distribuição dos profissionais conforme a função que desempenhavam em seu trabalho. Houve uma distribuição equilibrada entre as principais funções, com discreto predomínio de médicos (26\%). Para somente $10 \%$ da amostra, este era o seu primeiro trabalho. O tempo médio de trabalho na saúde foi de cinco anos (d.p.=5,8 anos) e o tempo médio total de trabalho foi de 16,4 anos (d.p.= 8,9 anos).

Pouco mais de metade dos entrevistados mantinha outro emprego além das UBS e dos CAPs (51\%) e um quarto precisou fal-

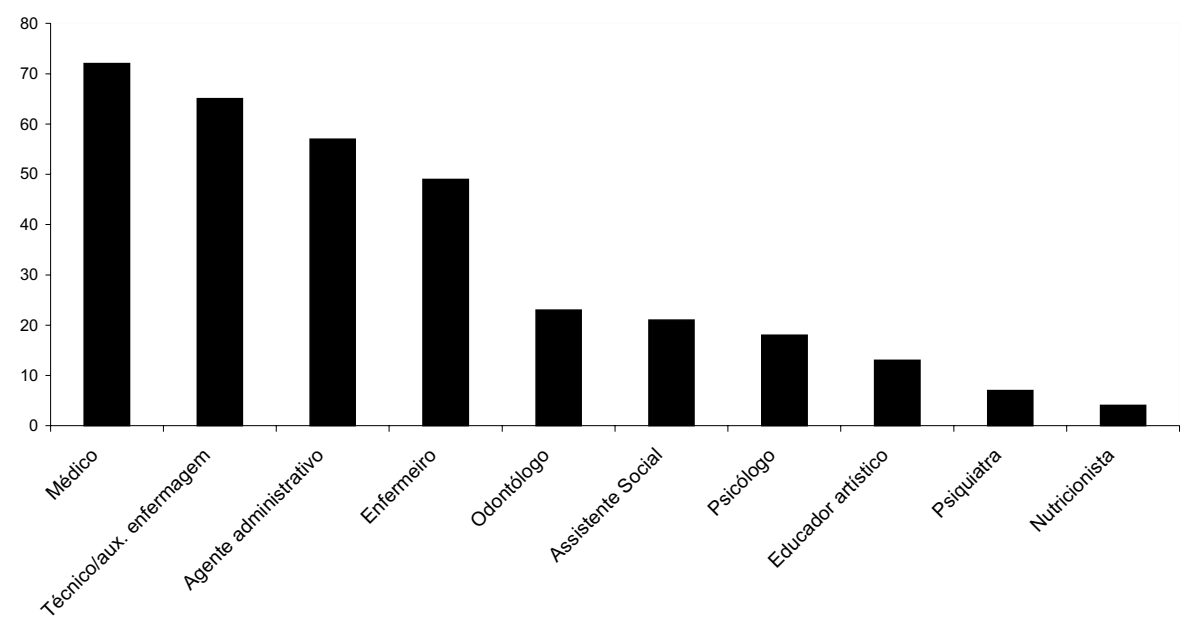

Figura 1 - Distribuição da amostra de profissionais de atenção básica à saúde conforme a função (n=329).

Figure 1 - Sample distribution of health professionals in primary healthcare according to job $(n=329)$. 
tar ao trabalho nos últimos 30 dias. As principais causas deste absenteísmo foram problemas pessoais de saúde (59\%) e problemas familiares (22\%). Em média, os trabalhadores atendiam cerca de 30 pessoas em um dia normal de trabalho, e a jornada de trabalho média, incluindo os demais empregos, era de 40 horas semanais.

As condições de trabalho foram avaliadas como inadequadas em $38 \%$ dos casos para o ambiente físico, em $46 \%$ para os aspectos relacionados às tarefas, em $34 \%$ para as relações institucionais e em $37 \%$ para as relações pessoais. Considerandose o ambiente físico, os itens mais referidos como inadequados foram a manutenção do prédio (67\%), o mobiliário (48\%) e a insuficiência de espaço (44\%). Nos aspectos relacionados às tarefas, destacaram-se a falta de recursos para o trabalho (83\%), a realização de tarefas muito variadas (65\%) e o excesso de trabalho burocrático (54\%). Quanto às relações institucionais, os destaques foram não estar de acordo com o andamento do trabalho (38\%), falta de reconhecimento pelo trabalho $(30 \%)$ e medo de ficar sem trabalho (19\%). Por último, os destaques referentes às relações pessoais foram não ver condições de progredir no trabalho $(49 \%)$, perda de tempo com outras tarefas (35\%) e pensar que seus erros podem afetar outras pessoas (19\%).

O hábito de fumar foi referido por $19 \%$ das pessoas e $61 \%$ não praticaram nenhuma atividade física regularmente no mês anterior à entrevista (Figura 2).

Problemas de saúde em geral foram referidos por $40 \%$ dos entrevistados, e os principais foram do aparelho circulatório (27\%) - com destaque para a hipertensão - e os osteomusculares (18\%). Nove por cento da amostra referiram mais de um problema de saúde (Figura 2).

Entre os que referiram problemas de saúde, $67 \%$ faziam uso de medicação regularmente e os medicamentos mais utilizados foram os anti-hipertensivos (34\%) e os antiinflamatórios (12\%). Independentemente de terem problema de saúde, $47 \%$ dos profissionais de saúde referiram uso de medicamentos nos últimos 15 dias, com destaque para os analgésicos (27\%). A automedicação era uma prática freqüente, pois um quarto dos entrevistados afirmou que a maioria dos medicamentos que usa é sem prescrição médica (Figura 2).

Quase a metade dos entrevistados (44\%) precisou consultar com médico nos três meses que antecederam a pesquisa. A maioria costumava utilizar serviços de saúde particulares ou de convênios, e somente $26 \%$ utilizavam o sistema público. Cinco por

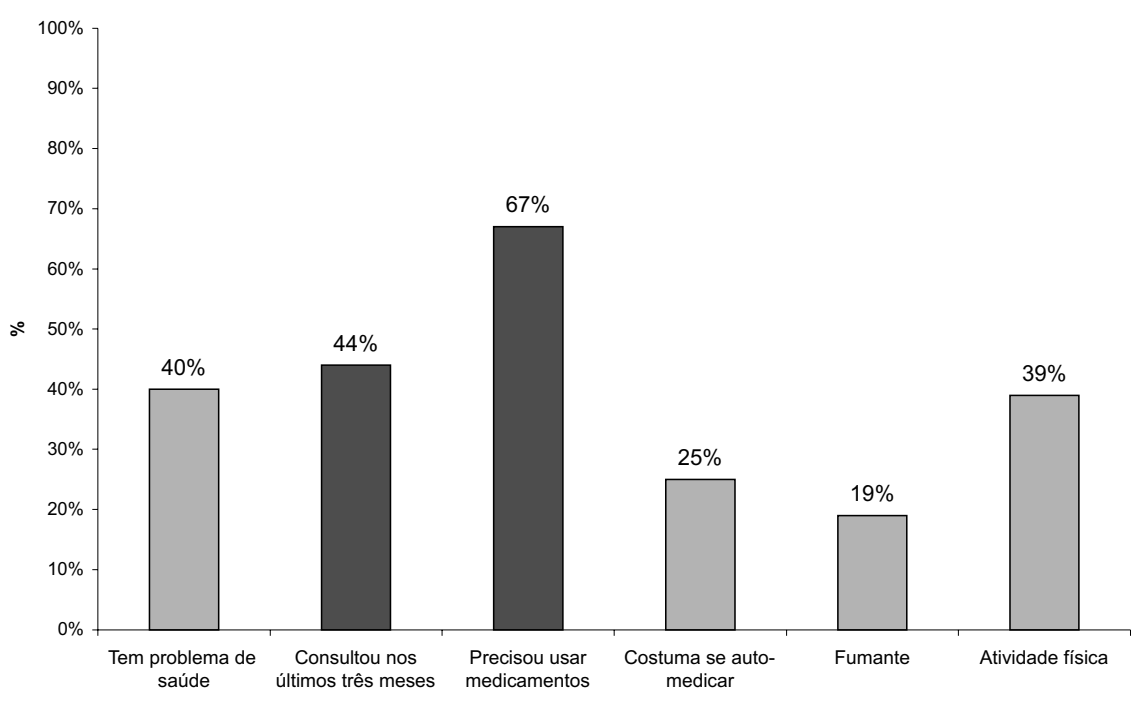

Figura 2 - Morbidade referida, automedicação e hábitos em saúde $(\mathrm{n}=329)$. Figure 2 - Reported morbidity, self-medication and health habits $(n=329)$. 
cento dos profissionais de saúde da rede básica apresentaram escores compatíveis com depressão de acordo o BDI.

A Figura 3 apresenta as prevalências de automedicação de acordo com a função, onde se destaca a magnitude deste desfecho entre os profissionais médicos. Comparados com os trabalhadores de nível médio, os médicos referiram quatro vezes mais o uso de medicamentos sem prescrição (Razão de Prevalências de 4,01 - IC 95\% 2,25-7,14). Associações igualmente significativas com automedicação foram encontradas para as variáveis escolaridade e classe social: quanto melhor a situação, maior a ocorrência de automedicação. Além disso, quem tinha mais de um vínculo empregatício referiu duas vezes mais automedicar-se do que quem só trabalhava na rede básica.

Quem realizava mais atendimentos por dia apresentou a menor prevalência de automedicação; no entanto, com exceção destes extremos, é possível evidenciar uma relação de dose-resposta.

\section{Discussão}

Em primeiro lugar, deve ser destacado o percentual de perdas e recusas do estudo $(18 \%)$, muito embora não tenham se concentrado em nenhuma categoria profissional, o que poderia, se ocorrido, con- figurar-se em um viés de seleção.

Foi possível delinear o perfil do profissional de saúde da atenção básica do município e conhecer sua percepção sobre as condições de trabalho. A maioria era do sexo feminino e um terço era de baixo nível socioeconômico.

A prevalência de automedicação encontrada em todos os profissionais foi de $24,8 \%$, similar à relatada por Loyola Filho ${ }^{11}$ em Minas Gerais, em estudo de base populacional realizado com moradores acima de 18 anos. Entretanto, foi inferior à prevalência descrita por Villarino e colaboradores em Santa Maria ${ }^{12}$ (53,3\%).

A maioria dos trabalhos publicados sobre o tema tem enfocado a automedicação em médicos ou em estudantes de enfermagem e medicina. Hem e cols. ${ }^{13}$ (2005), em estudo de coorte sobre automedicação em jovens médicos noruegueses, encontraram 54\% de prevalência entre o quarto e o quinto ano após a conclusão do curso, e entre os que usaram medicamentos no ano anterior à entrevista, 90\% foram autoprescritos. Estes achados são consistentes com a amostra estudada, onde $43 \%$ dos profissionais médicos referiram automedicação.

Dois inquéritos realizados na Finlândia ${ }^{14}$, em 1986 ( $n=2.671)$ e em 1997 ( $n=3.313)$, revelaram um aumento importante nas preva-

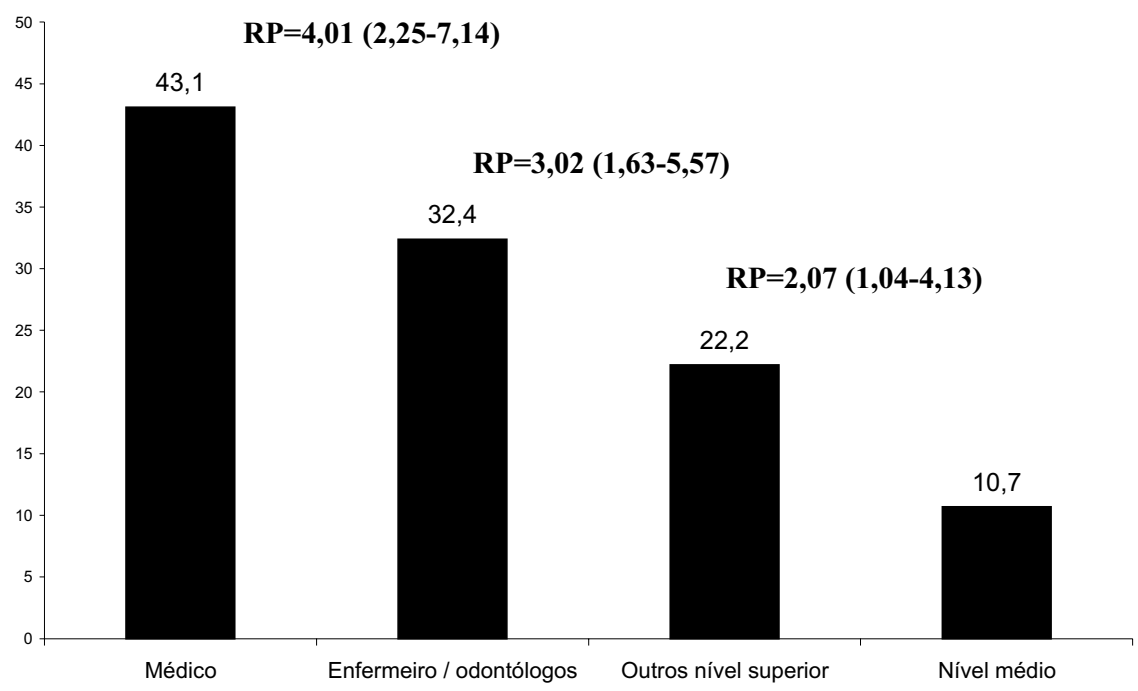

Figura 3 - Automedicação em profissionais de saúde conforme a função ( $n=329)$. Figure 3 - Self-medication in healthcare professionals according to job $(n=329)$. 
Tabela 1 - Distribuição dos profissionais de saúde em atenção básica de acordo com variáveis sociodemográficas e ocupacionais e prevalência de automedicação. Pelotas, RS, 2005.

Table 1 - Distribution of healthcare professionals in basic healthcare per sociodemographic and occupational data and prevalence of self-medication, Pelotas, RS, 2005.

\begin{tabular}{|c|c|c|c|c|}
\hline Variável & $\mathrm{n}$ & $\%$ & \% Automedicação & RP (IC95\%) \\
\hline \multicolumn{5}{|l|}{ Gênero } \\
\hline Masculino & 67 & 20,4 & 33,3 & $1,47(0,98-2,22)$ \\
\hline Feminino & 262 & 79,6 & 22,6 & 1,00 \\
\hline \multicolumn{5}{|l|}{ Idade (anos) } \\
\hline Até 30 & 48 & 14,6 & 31,3 & $1,44(0,72-2,87)$ \\
\hline 31 a 40 & 109 & 33,1 & 18,3 & $0,84(0,43-1,66)$ \\
\hline 41 a 50 & 124 & 37,7 & 29,0 & $1,34(0,72-2,47)$ \\
\hline 51 e mais & 48 & 14,6 & 21,7 & 1,00 \\
\hline \multicolumn{5}{|l|}{ Escolaridade } \\
\hline Médio & 99 & 30,1 & 13,3 & 1,00 \\
\hline Superior & 230 & 69,9 & 29,7 & $2,24(1,30-3,86)$ \\
\hline \multicolumn{5}{|l|}{ Classe social (ABIPEME) } \\
\hline$A$ & 44 & 13,4 & 34,1 & $1,84(1,02-3,34)$ \\
\hline B & 192 & 58,4 & 25,7 & $1,39(0,85-2,27)$ \\
\hline Ce D & 93 & 28,3 & 18,5 & 1,00 \\
\hline \multicolumn{5}{|l|}{ Local de trabalho } \\
\hline Unidade básica & 263 & 79,9 & 23,6 & 1,00 \\
\hline CAPs & 66 & 20,1 & 29,7 & $1,26(0,81-1,95)$ \\
\hline \multicolumn{5}{|l|}{ Tempo de trabalho (anos) } \\
\hline Até 9 & 97 & 30,1 & 25,8 & $1,17(0,71-1,91)$ \\
\hline 10 a 19 & 104 & 32,3 & 22,1 & 1,00 \\
\hline 20 e mais & 121 & 37,6 & 26,1 & $1,18(0,74-1,89)$ \\
\hline \multicolumn{5}{|l|}{ Tem outro emprego } \\
\hline Sim & 168 & 51,1 & 32,7 & $2,00(1,32-3,03)$ \\
\hline Não & 161 & 48,9 & 16,4 & 1,00 \\
\hline \multicolumn{5}{|l|}{ Absenteísmo último mês } \\
\hline Sim & 81 & 24,6 & 24,7 & 1,00 \\
\hline Não & 248 & 75,4 & 24,8 & $1,00(0,65-1,56)$ \\
\hline \multicolumn{5}{|l|}{ Atendimentos por dia } \\
\hline Até 10 & 46 & 14,0 & 23,9 & $2,03(0,89-4,66)$ \\
\hline 11 a 20 & 107 & 32,5 & 29,2 & $2,49(1,22-5,08)$ \\
\hline 21 a 40 & 108 & 32,8 & 29,0 & $2,46(1,20-5,04)$ \\
\hline Mais de 40 & 68 & 20,7 & 11,8 & 1,00 \\
\hline \multicolumn{5}{|c|}{ Condições de trabalho inadequadas $(*)$} \\
\hline Ambiente físico & 126 & 38,3 & 27,0 & $1,15(0,79-1,69)$ \\
\hline Tarefas & 152 & 46,2 & 23,7 & $1,09(0,74-1,59)$ \\
\hline Aspectos institucionais & 112 & 34,0 & 26,8 & $1,13(0,77-1,67)$ \\
\hline Aspectos pessoais & 120 & 36,5 & 30,3 & $1,40(0,96-2,04)$ \\
\hline Total & 329 & 100,0 & 24,8 & - \\
\hline
\end{tabular}

*A soma dos percentuais excede 100\% por tratar-se de quatro variáveis. As Razões de Prevalência têm como categoria de referência os que avaliaram as condições de trabalho adequadas para cada item.

* Total percentages exceed $100 \%$ because there are four variables. The reference category for Prevalence Ratios are those who assessed working conditions appropriate for each item

lências de automedicação entre médicos de ambos os sexos (de $28 \%$ para $44 \%$ entre os homens, e de $29 \%$ para $49 \%$ entre as mulhe- res). Médicos generalistas vinculados ao setor público tiveram um aumento de $31 \%$ para $49 \%$ no período. 
O aumento da automedicação entre pessoas com maior escolaridade já havia sido relatado por Vilarino ${ }^{12}$ e por Loyola Filho ${ }^{11}$. As categorias profissionais com o segundo maior índice de automedicação nesta amostra foram os enfermeiros e odontólogos (32\%). Encontram-se na literatura diversos trabalhos sobre automedicação entre estudantes de enfermagem, profissão que tem sido alvo de estudos sobre o impacto do estresse sobre o trabalho, evidenciando maiores riscos de automedicação ${ }^{16-18}$.

A curiosa relação entre número de atendimentos diários e automedicação pode ser explicada pelas características do processo de trabalho em atenção básica. Os profissionais de nível médio são responsáveis por atribuições administrativas, como, por exemplo, a recepção e o registro. Também de nível médio, os auxiliares e técnicos de enfermagem realizam ações com menor dispêndio de tempo, como aplicação de vacinas e aferições de peso, temperatura e pressão arterial.

A consistência entre estes achados sugere que os médicos, profissionais de melhor escolaridade e condição socioeconômica, devem merecer da gestão da saúde pública uma atenção especial. Esta categoria, exposta ao estresse do trabalho, geralmente com mais de um vínculo empregatício e com fácil acesso às substâncias, pode estar subestimando os perigos da automedicação, identificada como um dos fatores de risco para o abuso de substâncias psicoativas entre profissionais de saúde na Espanha ${ }^{15}$.

Acredita-se que este é um tema que deve ser abordado ainda nas escolas formadoras de profissionais de saúde, alertando para os riscos da automedicação e focalizando suas causas e aspectos ocupacionais, passíveis de modificação através de políticas e programas de valorização profissional e de oferta de melhores condições de trabalho.

\section{Referências}

1. Nemes Filho A. A unidade básica e o sistema de saúde. In: Schraiber LB, Nemes MIB., Mendes-Gonçalves RB, orgs. Saúde do adulto, programas e ações na Unidade Básica. São Paulo: Hucitec; 1996. p. 276-86.

2. Fassa AG, Facchini LA, Dall'agnol MM. Trabalho e morbidade comum em indústria de celulose e papel: um perfil segundo setor. Cad Saúde Públic 1996; 12(3): 297307.

3. Faria NM, Facchini LA, Fassa AG, Tomasi E. Processo de trabalho rural e saúde na serra gaúcha: um estudo descritivo. Cad. Saúde Pública 2000; 16(1): 115-28.

4. Palácios M, Duarte F, Camara VM. Trabalho e sofrimento psíquico de caixas de agências bancárias na cidade do Rio de Janeiro. Cad Saúde Pública 2002; 18(3): 843-51.

5. Kuchenbecker R. Exposição ocupacional a sangue e secreções corporais no Sistema Único de Saúde em Porto Alegre: epidemiologia e políticas de prevenção [dissertação de mestrado]. Pelotas: Universidade Federal de Pelotas, RS; 1999.

6. Pedrosa JIS, Teles JBM. Consenso e diferenças em equipes do Programa Saúde da Família. Rev Saúde Pública. 2001; 35(3):303-11.
7. Amaral MA. Atenção à saúde mental na rede básica: estudo sobre a eficácia do modelo assistencial. Rev Saúde Pública 1997; 31(3): 288-95.

8. Paulo LG, Zanine AC. Automedicação no Brasil. Rev Ass Med Bras 1988; 34: 69-75.

9. Figueroa NL, Schufer M, Muinos R. et al. Um Instrumento para a Avaliação de Estressores Psicossociais no Contexto de Emprego. Psicol Reflex Crit 2001; 14(3): 653-9.

10. Andrade L, Gorenstein C, Vieira Filho AH, Tung TC, Artes R. Psychometric properties of the Portuguese version of the State-Trait Anxiety Inventory applied to college students: factor analysis and relation to the Beck Depression Inventory. Braz J Med Biol Res. [periódico na Internet]. 2001 Mar [citado 2006 Out 08]; 34(3): 367-374. Disponível em: http://www. scielo.br

11. Loyola Filho AI, Uchoa E, Guerra HL; Firmo JOA, LimaCosta MF. Prevalência e fatores associados à automedicação: resultados do projeto Bambuí. Rev Saúde Pública 2002; 36(1): 55-62.

12. Vilarino JF, Soares IC, Silveira CM, Rödel APP, Bortoli R, Lemos RR. Perfil da automedicação em município do Sul do Brasil. Rev Saúde Pública 1998; 32(1): 43-9. 
13. Hem E, Stokke G, Tyssen R, Gronvold NT, Vaglum P, Ekeberg O. Self-prescribing among young Norwegian doctors: a nine-year follow-up study of a nationwide sample. BMCMed 2005; 3: 16.

14. Töyry S, Räsänen K, Seuri M, Aärimaa M, Juntunen J, Kujala S, Husman K. Increased personal medication use among Finnish physicians from 1986 to 1997. Br J Gen Pract 2004; 54(498): 44-6.

15. Sanz Yagüez F, López Corbalán JC. Abuso de sustancias psicoactivas entre los profesionales de la salud. Rev Esp Anestesiol Reanim 1999; 46(8): 354-8.
16. Magaldi L, Rocafull, J. Farmacovigilancia y hábitos de consumo de medicamentos en los estudiantes de la Escuela de Enfermería de la Universidad Central de Venezuela. Rev Fac Med (Caracas) 2004; 27(1): 74-8.

17. Alvitres BC, Bejarano IF. Autodiagnóstico y automedicación en el personal de enfermería: una práctica usual en nuestros días. Temas Enferm Actual 2002; 10(49): 37-40.

18. Lautert L, Chaves EHB, Moura GMSS. O estresse na atividade gerencial do enfermeiro. Rev Panam Salud Publica 1999; 6(6); 415-25.

Recebido em: 05/06/06

Versão final reapresentada em: 08/10/06

aprovado em: 10/12/06 